\title{
Brain-Computer Interfaces on Track to Home: Results of the Evaluation at Disabled End-Users' Homes and Lessons Learnt
}

\author{
Felip Miralles ${ }^{1 *}$, Eloisa Vargiu ${ }^{1}$, Xavier Rafael-Palou ${ }^{1}$, Marc Solà ${ }^{1}$, Stefan Dauwalder ${ }^{1}$, \\ Christoph Guger ${ }^{2}$, Christoph Hintermüller ${ }^{2}$, Arnau Espinosa ${ }^{2}$, Hannah Lowish ${ }^{3}$, \\ Suzanne Martin ${ }^{4}$, Elaine Armstrong ${ }^{4}$ and Jean Daly ${ }^{4}$ \\ ${ }^{1}$ Barcelona Digital Technology Center, Barcelona, Spain, ${ }^{2}$ g.tec Medical Engineering GmbH, Schiedlberg, Austria, \\ ${ }^{3}$ Telehealth Solutions, Medvivo Group, Wiltshire, UK, ${ }^{4}$ The Cedar Foundation, Belfast, UK
}

\section{OPEN ACCESS}

Edited by:

Nadia Bianchi-Berthouze, University College London, UK

Reviewed by:

Fabien Lotte,

National Institute for Research in Computer Science and Control,

France

Pascual Gonzalez,

University of Castilla-La Mancha,

Spain

${ }^{*}$ Correspondence:

Felip Miralles

felip.miralles@eurecat.org

Specialty section:

This article was submitted to

Human-Media Interaction,

a section of the journal Frontiers in ICT

Received: 03 July 2015 Accepted: 03 November 2015 Published: 25 November 2015

Citation:

Miralles F, Vargiu E, Rafael-Palou X

Solà M, Dauwalder S, Guger C, Hintermüller $C$, Espinosa A, Lowish $H$,

Martin S, Armstrong $E$ and Daly $J$ (2015) Brain-Computer Interfaces on

Track to Home: Results of the

Evaluation at Disabled End-Users

Homes and Lessons Learnt.

Front. ICT 2:25.

doi: 10.3389/fict.2015.00025
The BackHome system is a multifunctional $\mathrm{BCl}$ system, the final outcome of a UserCentered Design approach, whose ambition is to move $\mathrm{BCl}$ systems from laboratories into the home of people in need for their independent home use. The paper presents the results of testing and evaluation of the BackHome system with end-users at their own homes. Results show moderate to good acceptance from end-users, caregivers, and therapists, which reported promising usability levels, good user satisfaction, and levels of control in the use of services and home support based on remote monitoring tools.

Keywords: brain-computer interface, practical electrodes, user-centered design, telemonitoring and home support, assistive technology, cognitive rehabilitation, teleassistance, end-user evaluation

\section{INTRODUCTION}

An important number of research projects in the last number of years have contributed to improve brain-computer interface (BCI) technologies and a number of different applications for this alternative means of human-computer interaction have been produced (Lynch, 2002; Kaufmann et al., 2013). In the roadmap toward 2020 for BCI research proposed by Brunner et al. (2015), applications are targeted at replacing, restoring, or improving the functions of people with some degree of disability as a key objective of future BCI research and innovation. Additionally, BCI use by ablebodied users for enhancing their functions or for broadening their leisure activities is expected to gain momentum and drive research.

The progress of BCI research within those "replace" and "restore" scenarios aim to deliver BCIbased products that represent an alternative to current assistive technologies (AT). Additionally, the progress of $\mathrm{BCI}$ research within the "improve" scenario will deliver new rehabilitation methods and tools. The BackHome project ${ }^{1}$ has promoted these trends with the ambition to move BCI systems from laboratories and controlled environments into the home of people for their independent use. A number of recent studies have indicated that BCI could be a useful solution for target end-user; however, these studies have also been in controlled environment or laboratory based (Nijboer et al., 2008; Holz et al., 2013; Schreuder et al., 2013; Zickler et al., 2013). User-Centered Design (UCD) has been developed as a way of reducing this gap between the laboratory and the real world use of $\mathrm{BCI}$

\footnotetext{
${ }^{1}$ www.backhome-fp7.eu/
} 
(Kübler et al., 2014). Recently, case studies have started to challenge these barriers and move $\mathrm{BCI}$ into the domestic environment (Vaughan et al., 2006; Sellers et al., 2010; Holz et al., 2015). Limitations to home-based testing include the cost of the systems, the difficulty of the set up, low response rate, the dependence on motivated caregivers, the need for technical support, the complexity of the software and hardware, and the ethical implications. Kübler et al. (2015) observed that because of the challenges associated with home-based evaluations, it is likely that numbers of endusers taking part will remain small. These studies have started to unpick the real world challenges that face BCI when they are introduced to more complex environments; however, further work is necessary to explore the realities of home use, and how that technology can be incorporated into the lives of the user and their caregiver. Caregivers play a key role in bringing BCI toward home-based solutions.

Following on from the work of Holz et al. (2015), we incorporate a multimodal system aimed at moving BCI into end-user homes and their non-expert caregivers. In particular, we integrated the research in practical electrodes, easy-to-use software, and home support aids into an innovative multimodal AT, which provides useful services for communication, Web access, cognitive rehabilitation, leisure, and environmental control with nonexpert support. Miralles et al. (2015) illustrated the framework adopted in the design and development of the BackHome system incorporating a UCD approach. In this paper, we assess the enduser's satisfaction with this unique BCI-based AT, presenting and discussing the results from the home evaluation of the system with people living with acquired brain injury. To the best of our knowledge, this is the first time a BCI with such a wide range of functionalities has been evaluated at home by end-users. We also explore the experiences of caregivers and therapists in their role to facilitate end-users operate the BCI system. To the best of our knowledge, no research has explored the perspectives of nonexpert caregivers in the set up and support of end-users using BCI at home.

\section{MATERIAL}

The system development has been iterative and incremental to trigger reflections and get feedback from end-users, their families, caregivers, and therapists prior to the implementation of the final system [see Kübler et al. (2014)]. A User-Centered Design (UCD) approach has been adopted at each phase of the system definition and implementation in order to take into account users' feedback to have a solution that reflects users' requirements, needs, and preferences. In summary, the approach consisted of three main steps: (1) user requirements gathered through focus groups with end-users, caregivers, and therapists; (2) users' evaluation to improve the system according to an evolutionary prototyping approach that takes into account continuous feedback from standalone and integrated testing (Daly et al., 2015a,b); and (3) the final system deployment.

Driven by this UCD approach toward independent home use, BackHome developed and refined five key innovations advancing current state of art: (i) a modular and distributed architecture able to meet the requirements of a multifunctional BCI with remote home support (see Figure 1); (ii) BCI equipment with practical electrodes aimed at setting a new standard of lightness, autonomy, comfort, and reliability; (iii) easy-to-use software tailored to people's needs to manage a complete range of multifunctional applications finely tuned for one-click command and adaptive usage; (iv) a telemonitoring and home support system to remotely monitor and assist BCI independent use; and (v) a Webbased application for therapists, which offers remote services to plan and monitor BCI-based cognitive rehabilitation and pervasively assess the use of the system and the quality of life of the individual.

A new biosignal acquisition system called g.Nautilus was developed [see Hintermüller et al. (2015)] and is shown in Figure 2. Its biosignal amplifier uses wireless technology to transmit the EEG signals with 24 bit resolution. The signal of each EEG channel is highly oversampled in order to keep the signal-to-noise ratio (SNR) high at the offered rates of 250 and $500 \mathrm{~Hz}$. Furthermore, it is capable of measuring the electrode-skin impedance at each electrode position for both gel-based and dry electrodes. A base station which is connected to the host system through USB is used to receive the recorded and digitized EEG signals. The biosignal amplification unit consists of the headset including the wireless biosignal amplifier electrodes, an EEG cap, and the base station including a USB cable for connecting it to the host computer and a QI compatible wireless charging station. The 34 or 10 electrodes caps including reference channel and ground are preconnected to the amplifier using a preconfigured set of electrode positions. The BCI user interface is based upon the Screen Overlay Control Interface (SOCI) library (Kapeller et al., 2013), which allows embedding the BCI stimuli on top of the native interface of any user application and communicates with the BCI hardware via a network connection. Masks, which are smaller, are automatically centered by SOCI within the visible area. Alternatively, the new version of the Application ConTrol and Online Reconfiguration (ACTOR) protocol, described by Hintermüller et al. (2013), allows the application to explicitly position its masks on the screen. An easy-to-operate user interface was developed for the non-expert caregiver, shown in Figure 3, that enables a person to start the system with just one-click, to create the classifier (in training mode), and to shutdown the BCI system. Furthermore, when the system is started, it automatically activates the check signals mode and starts the signal acquisition. This simplifies the mounting of the electrode cap as the signal quality display is visible during the whole procedure.

The system offers a set of innovative, flexible, and extensive services (Käthner et al., 2014), partly shown on the screens of Figure 2: Smart Home control, cognitive stimulation, and Web access. Smart Home control service is aimed at giving control over the environment, as well as over useful devices (e.g., a light, a fan, and a radio) as well as to interact with the XBMC multimedia player. Cognitive stimulation services allow users to improve their cognitive capabilities by performing cognitive rehabilitation tasks assigned by a therapist; i.e., Activities of Daily Living, Find a Category, and Memory (Vargiu et al., 2014), or by using their creative skills through Brain Painting (Münssinger et al., 2010). Web access services offer users the possibility to engage in social 


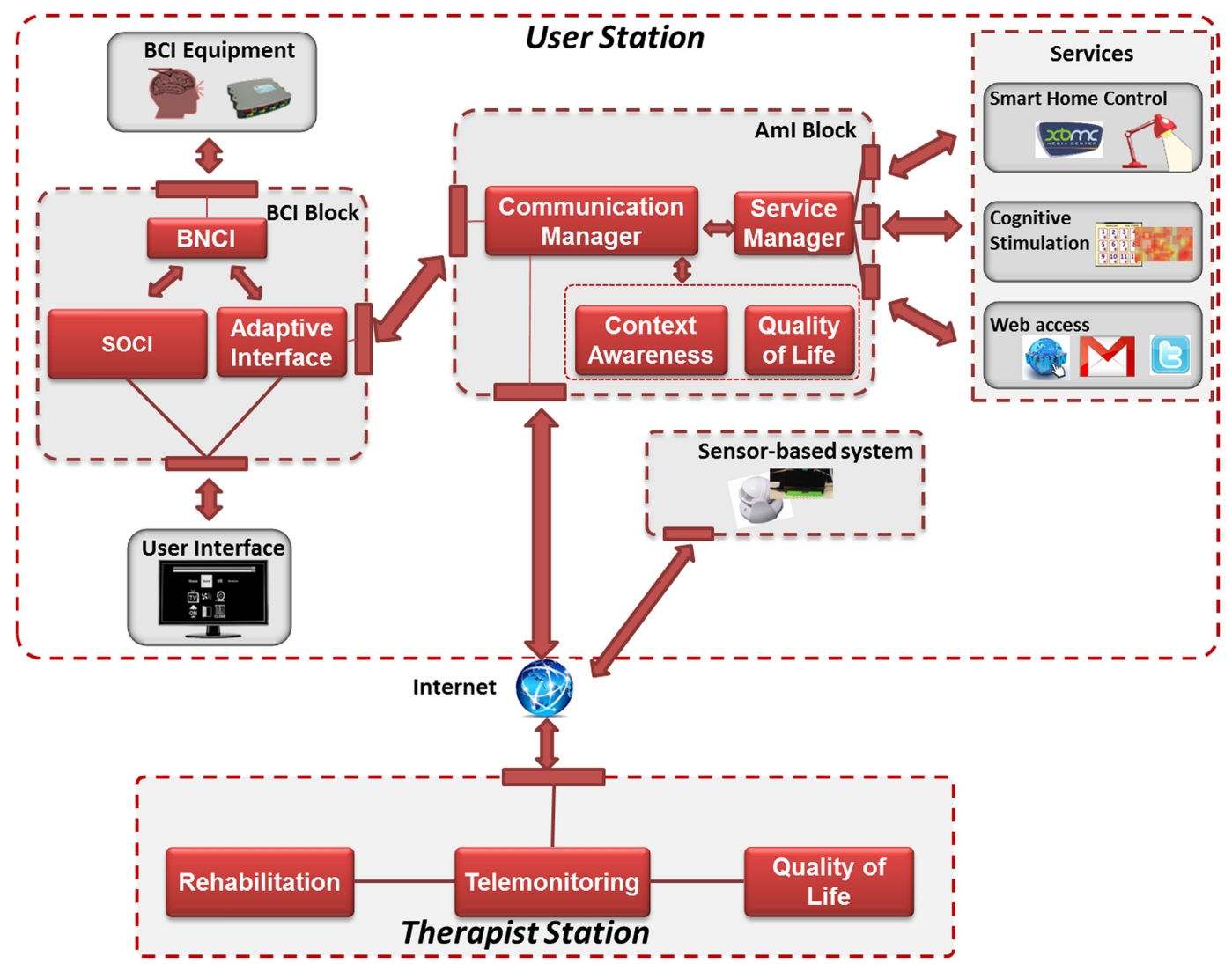

FIGURE 1 | The modular and distributed architecture
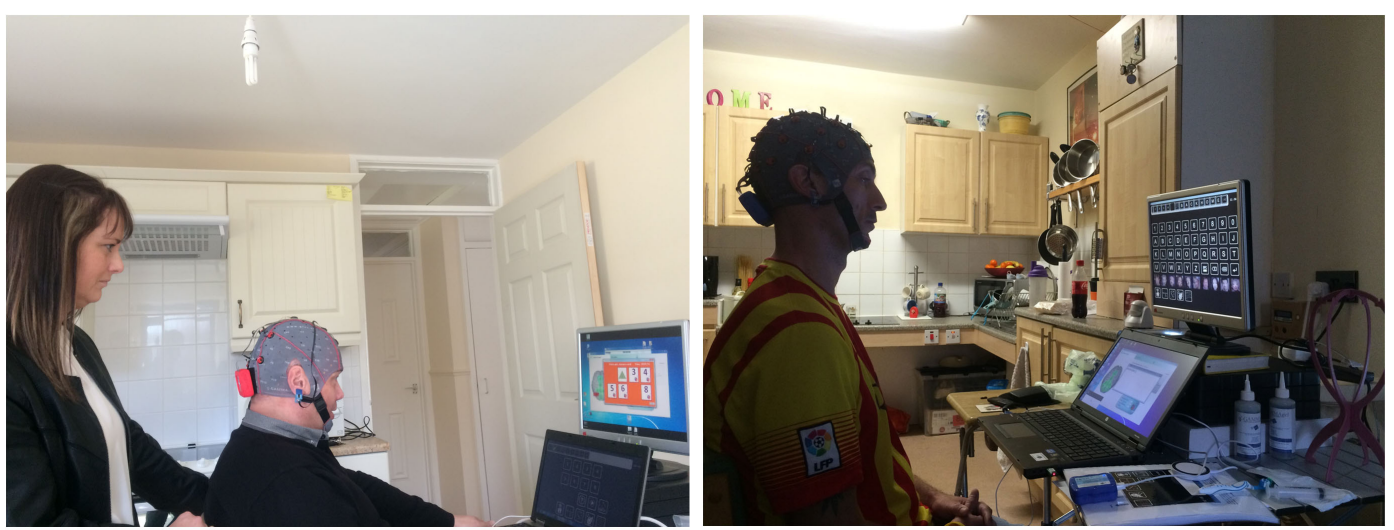

FIGURE 2 | End-users at their homes: Home User 1 (HU1, on the right) and Home User 3 (HU3, on the left) using the system

interaction through the Web, such as Web browsing, emailing, and social media. All the services rely on a P300 spelling and control system. In the adopted speller, the highlighting happens with freely selectable images (famous faces) instead of just changing the color of the background (Kaufmann et al., 2013). Users interact with the system through two separated screens: one for the BCI matrix and one for the selected service. The dynamic stopping method is an algorithm integrated into the software specifically with home use in mind whose purpose is to increase the selection speed by determining the optimal number of sequences during usage and dynamically making a selection (stopping) when a probability threshold is reached. Furthermore, it minimizes the number of false selections by suppressing unintended selections (if the user is not looking at the screen, for instance). The system also includes a sensor-based telemonitoring and home support system that is aimed at recognizing habits of the end-user, assessing her quality of life, and capture statistics about the BCI usage. The system is composed of a network of wireless environmental 

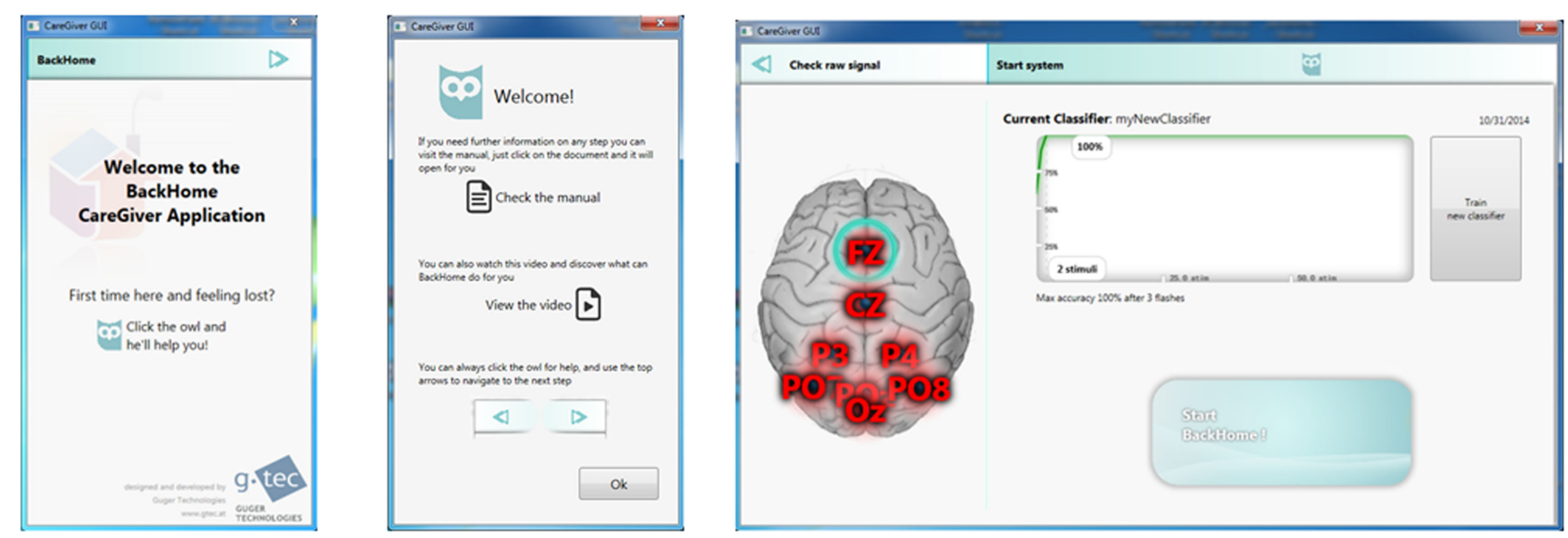

FIGURE 3 | The caregiver interface. Three screenshots of the welcome window (on the left), the help window with links to the manual and a video (in the middle), and the signal quality window with a plot of the trained classifier (on the right).

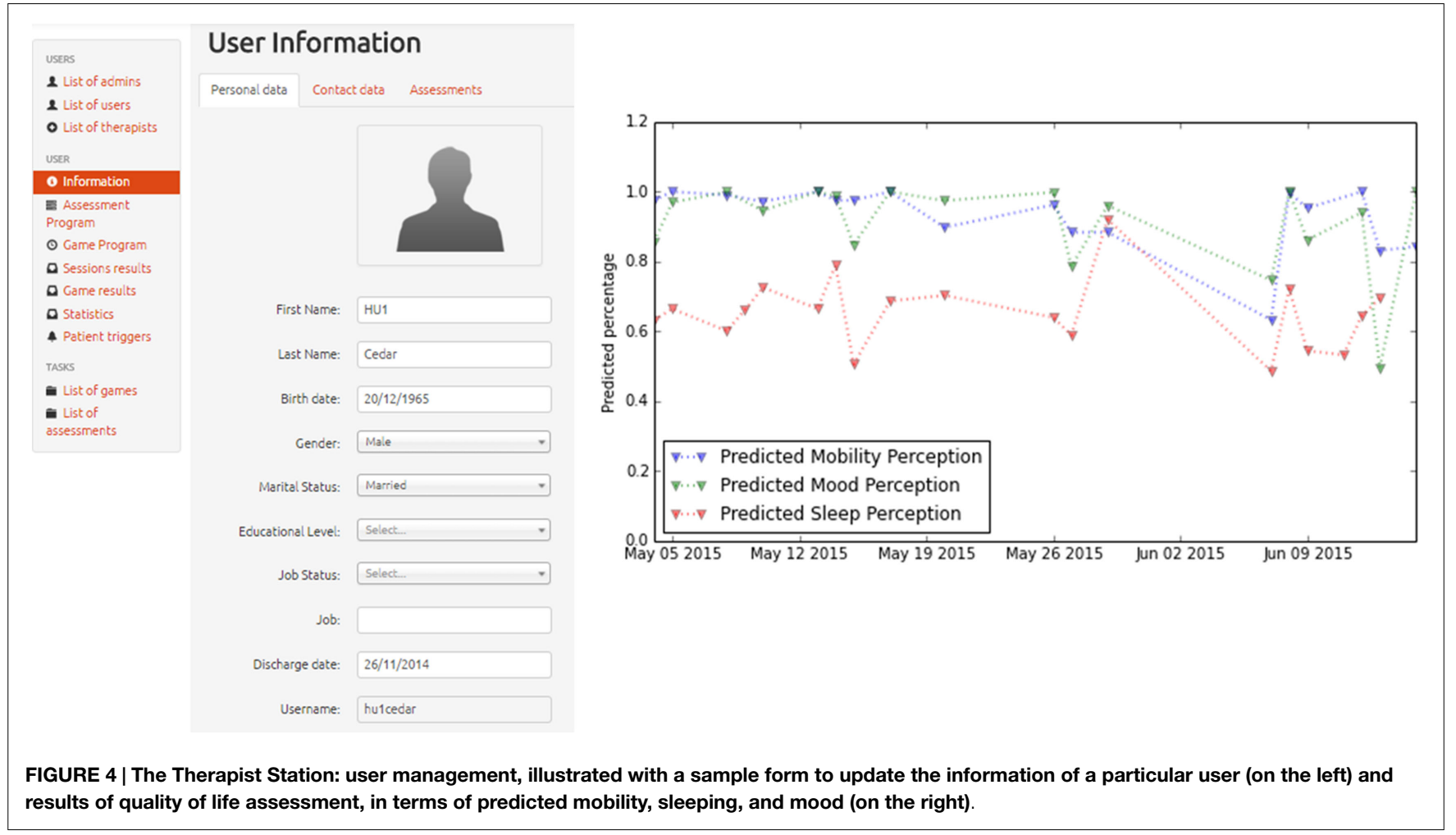

sensors connected to a Raspberry pi-based central unit ${ }^{2}$ for indoor monitoring. Moreover, the end-user's smartphone, in which the Moves app ${ }^{3}$ has been installed, is used to monitor outdoor activities. The system is able to continuously gather data from the room in which the user is spending her time when she is at home, to recognize and study the end-user habits (Rafael-Palou et al., 2015), and to assess user's quality of life (Vargiu et al., 2015).

${ }^{2}$ https://www.raspberrypi.org/

${ }^{3} \mathrm{http}: / /$ www.moves-app.com/
A Web application called the Therapist Station (see Figure $\mathbf{4}^{4}$ ) provides therapists the ability to manage their clients remotely, assign cognitive rehabilitation tasks, undertake quality-of-life assessment, and enhance communication between therapist and the end-user using the BCI. Therapists are able to interact with the users remotely in real time or asynchronously and monitor the use of the BCI-based AT, the outcomes of the cognitive rehabilitation tasks and the assessment of quality of life. It enables the therapist

${ }^{4}$ For the sake of anonymity, the real name has been omitted and the user has been named "HU1 Cedar." 
to plan, schedule, telemonitor, and personalize the prescription of cognitive rehabilitation tasks and quality-of-life questionnaires using the therapist station and to motivate the user to carry out those tasks inside her therapeutic range (i.e., supporting her progress), in order to help the user attain beneficial therapeutic results.

\section{METHODS}

A total of 4 participants were recruited for the 6-week homebased evaluation of the system. For the sake of anonymity, they will be referred as Home User 1 (HU1), Home User 2 (HU2), Home User 3 (HU3), and Home User 4 (HU4). Only two of the participants concluded the 6-week evaluation period. Attempts to set up and keep the system up and running for the 6 weeks in HU2's home failed due to unstable Internet connection and other basic technological barriers of his residential setting with shared resources, whereas HU4 became ill after the installation of the $\mathrm{BCI}$ and did not recover in time to participate in the home-based testing. Thus, the evaluation has only been performed with HU1 and HU3.

HU1 and HU3 are 50 and 37 years old, respectively. They experienced a severe brain injury as a result of a car accident (in 2001, HU1, and in 2004, HU3), are currently living in the community postacute service rehabilitation, and have been involved in the BackHome project from the beginning. They had no previous experience of BCI prior to the project. The sensors were installed in their home and Moves was downloaded onto their smartphone. They answered the questionnaire needed to test the telemonitoring system each day. HU1 used a g.Nautilus with 8 gel electrodes that were fixed in a medium size cap, whereas HU3 used a gel-based g.Nautilus with 32 gel electrodes that were fixed in a medium size cap. In both cases, the data from the eight channels (Fz, Cz, Pz, P3, P4, PO7, PO8, and Oz) along with a reference attached to the right ear and a ground at $\mathrm{FPz}$, was amplified by a waterproof device with contactless charging attached by velcro to the back of the cap and the signals were wirelessly transmitted to the base station. In order to support the HU3's non-expert caregiver, the 8 electrodes that required gel were highlighted on the cap with a small strip of brown tape. The laptop recording the data and displaying all of the BCI's applications was placed in front of both users. A computer stand was placed behind the laptop to support the external monitor to be visible above the laptop. The P300 matrix was visible on the laptop and the external monitor displayed the systems applications. The BCI user interface was configured in both cases like this: $120 \mathrm{~ms}$ for the display time of the famous faces icons, $80 \mathrm{~ms}$ for the time in between flashes, and 10 for the number of flashes before a selection.

A training session on the set up of the BCI using gel-based electrodes was run for the 4 caregivers. ${ }^{5}$ The session involved a thorough explanation of their role in the project and a step-by-step demonstration of how to set up the system and the functionality of each application. Each attendee then got the opportunity to set up a user on the system. The caregivers received an easy-to-use

${ }^{5}$ For the sake of anonymity, they will be referred as Caregiver 1 (CG1), Caregiver 2 (CG2), Caregiver 3 (CG3), and Caregiver 4 (CG4). manual to support them during the evaluation. At least two additional training sessions were undertaken when the system was deployed into the participant's home and shadowing sessions were completed.

A folder was given to each home user and caregiver to outline their tasks and support them through the 6 weeks of testing. Each week, the end-user was asked to complete at least three tasks: 1 set task to demonstrate use on an aspect of the system, 1 cognitive rehabilitation task sent to the user via the therapist station, and 1 free usage task where users could do anything they like. Endusers specified their preferred tasks during testing of the previous iteration of the prototype and these were incorporated into the set tasks. The set tasks were tweeting BCI at Home, viewing a clip on YouTube, using the multimedia player, posting a comment on Facebook about the weather, Google the search term BCI, perform cognitive rehabilitation sessions attempting each of the three tasks (starting at level 1 and increasing to level 2 and 3), and create a picture using the BrainPainting application. The task was deemed complete when each step that had been set out by the research team was performed on the system and this was self-reported by the end-user/caregiver.

Several meetings were organized with specialist professional groups to gather perspectives on the BackHome therapist station and to identify its usability in a real life setting. The professionals involved in this evaluation were primarily occupational therapists $(N=44)$. Other healthcare professionals included psychologists $(N=3)$, speech and language therapist's $(N=2)$, physiotherapist's $(N=2)$, a social worker $(N=1)$, and a nurse $(N=1)$.

The Ulster University Ethics Committee provided ethical approval for all the aspects related to the end-users (which come from vulnerable groups) and caregivers, including selection, recruitment, informed consent, training, testing, and reporting (both text and multimedia).

\section{RESULTS}

\subsection{End-Users' Satisfaction}

At the end of the evaluation, end-users were asked to complete the NASA-TLX [NASA-Task Load Index by Hart and Staveland (1988)], the eQUEST 2.0 [extended Quebec User Evaluation of Satisfaction with Assistive Technology by Demers et al. (2002)], and a customized usability questionnaire. Results reported by HU1 ad HU3 are illustrated in Table 1. Both users reported

${ }^{6}$ Range $1=$ not satisfied at all to $5=$ very satisfied.

TABLE 1 | Results from satisfaction questionnaires.

\begin{tabular}{lccc}
\hline & HU1 & HU3 & Max. score \\
\hline eQUEST 2.0 total score & 4.15 & 4 & 5 \\
eQUEST 2.0 added items & 3.7 & 3.7 & 5 \\
NASA-TLX & 66.61 & 37 & 100 \\
Did you feel in control, while using the system? & Yes & No & $\mathrm{n} / \mathrm{a}$ \\
Would you describe the system as intuitive? & Yes & Yes & $\mathrm{n} / \mathrm{a}$ \\
Operating the interface was & Easy & Easy & $\mathrm{n} / \mathrm{a}$ \\
Did you like the symbols/icons of the interface? & Yes & Yes & $\mathrm{n} / \mathrm{a}$ \\
Did you like the colors of the interface? & Yes & Yes & $\mathrm{n} / \mathrm{a}$
\end{tabular}


satisfaction on the eQUEST 2.0 with a slightly lower satisfaction rating on the BCI specific added items. HU1 rated ease of use, effectiveness, and reliability as most important on eQUEST 2.0, and HU3 reported safety, speed, and reliability as the most important. HU1 reported on the eQUEST 2.0 that the wireless headset on the g.Nautilus was the only way to have it although the cap needs to be more stylish. In terms of the AT ease of use, he felt that the two screens make it difficult to concentrate. On the eQUEST 2.0, HU3 felt the ease of adjusting was temperamental and inconsistent, the ease of use was inconsistent and the speed was sometimes good, sometimes not so good. HU1 reported far higher workload on the NASA-TLX compared to HU3. HU1 reported feeling frustrated at times during the evaluation and this effort to control the system could be reflected in the NASA-TLX score. $\mathrm{HU} 3$ reported not feeling in control when operating the BCI on the usability questionnaire.

After each session was complete, the therapist station would automatically open up on the laptop and ask the user to answer, How satisfied were you with the BCI session? (with 10 being very satisfied and 0 is not satisfied) and How would you estimate your level of control was over the BCI during the last session (did the BCI respond to the selections you wanted to make) and the user was asked to selection from: High (100-90\%); Medium (90-70\%); Low (70-50\%); and Zero (50-0\%). Additionally, following the set task and cognitive rehabilitation task the end-user was asked, Could you complete the task? yes/no. The BCI system was set up in the kitchen of HU1's home (see Figure 2, left side). HU1 reported an average satisfaction of 6.81 ranging from 0 to 10 over the 6-week evaluation (Figure 5, left side). ${ }^{7}$ The variation in satisfaction was linked to BCI performance according to the comments left on the therapist station not a good session because of the inability for the system to be responsive. HU1 had a mixture of positive and negative experiences ranging from I had to make the selections twice on most occasions to Great session. Probably the best yet. Additional, HU1's perceived level of control was medium and that the system responded to his desired commands sometimes to mostly. The

\footnotetext{
${ }^{7}$ The 0 scores on May 1 and May 30 were set by error and were excluded from the average score.
}

caregiver supported the user to navigate the system and complete all but 4 tasks. They reported a satisfaction rate of 8.61 out of 10 with the set up of the system. HU3 rated his satisfaction with the system at 5.84 out of 10 indicating he was more or less satisfied (see Figure 5, right side). He rated his level of control operating the BCI as low to medium and that the system responded to his desired commands sometimes to mostly. HU3 reported that activities of "Activity of Daily Living" level 2 were the only task for which he did not understand the commands. There also was difficulty when the Twitter page would not come up. HU3 enjoyed the experience when it was very reactive describing operating the BCI as mentally rewarding and fun.

During the 6-week evaluation all of the set tasks, cognitive rehabilitation tasks and free usage tasks were attempted by HU1. HU1 was able to successfully complete $72 \%$ (13 out of the 18) of the tasks set out by the research team. The reported reason for not completing the tasks was the inability to make a selection due to frequency of suppressed selections. This means that the threshold for the system to make a selection was not reached so a selection was not made and the flashing continued. The caregivers recorded the various applications explored during the free usage sessions, which were Google searches, using the multimedia player, social media, YouTube, and controlling home environment. It is inferred that a number of other activities were undertaken on the BCI as 5 additional cognitive rehabilitation sessions were found on the therapist station having been completed during the evaluation but were not accounted for within the folder. HU1 reported that the web tags to make selection were placed over the text field he was trying to type into obscuring his view. HU3 lived in an apartment and the BCI system was set up in the living area that was connected to his kitchen (see Figure 2, right side). HU3 was able to complete $61 \%$ (11 of the 18) of the tasks completely during the 6-week evaluation. The caregiver recorded the tasks which end-users tried to complete during free usage, which included free spelling, cognitive rehabilitation, social media, YouTube, BrainPainting, and interacting with his home environment. In keeping with the experiences of HU1, HU3 completed 6 additional cognitive rehabilitation tasks during the evaluation that were not reported by the caregiver but were recorded on the therapist station.

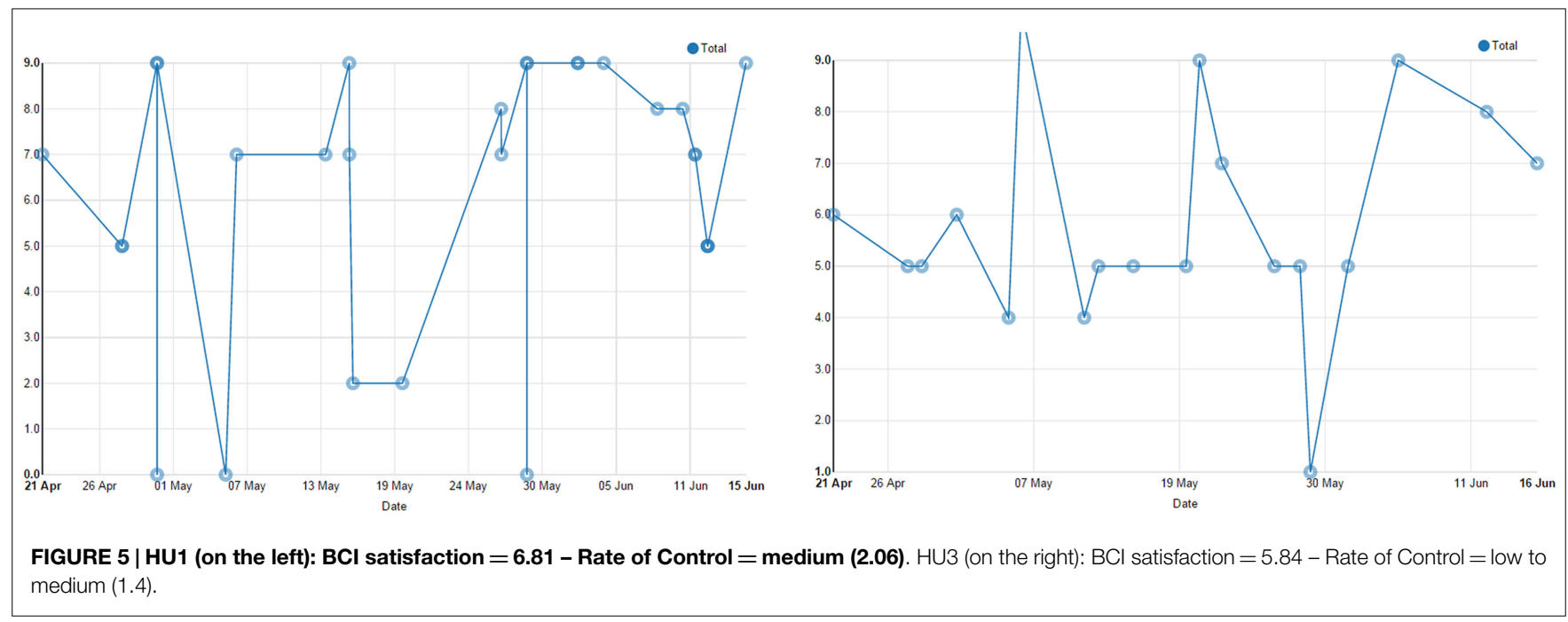




\subsection{Caregivers' Satisfaction}

The 4 caregivers were required to set up the end-user to operate the BCI on three occasions during each of the 6 weeks and complete a daily set up questionnaire after each of the 18 sessions. After the training sessions, and after the 6 weeks home testing (two out of four), caregivers were also asked to complete a satisfaction questionnaire; Table 2 depicts the questionnaire and the summary of results.

\subsection{Therapists' Satisfaction}

A protocol was defined to test the Therapist Station which involved the following groups of tasks: user management; user assessment; Cognitive Rehabilitation scheduling; BCI usage statistics; and triggers configuration. After following the protocol, therapists were asked to fill a questionnaire composed of 43 questions about all the actions performed during the testing. A total of $N=50$ therapists felt the BackHome platform could benefit their clients and equally, $N=50$ believed the BackHome platform could benefit their day-to-day practice. The functionality, usability, and application to practice were aspects of the system that therapists liked. In particular, the "measures of progress," "cognitive rehabilitation," and "quality of life measures" were particularly useful for therapists. There were a number of suggestions made during the evaluation. Overall, the therapist station was viewed in a positive light and considered to be an asset to daily practice. On average, the $36.63 \%$ of the therapists evaluated as positive (4) the overall platform and the $44.22 \%$ as very positive (5), making a total of $80.86 \%$ of positive and very positive evaluation.

\section{DISCUSSION}

To the best of our knowledge, this is the first time a multifunctional BCI with such a wide range of services has been evaluated at home by end-users, also taking the non-expert caregivers' perspective into account. Participants were enthusiastic about their experience evaluating the BackHome system in their own home. Ultimately, there were challenges; however, the learning from this evaluation is essential to realize the fundamental goal of moving BCI into peoples' homes as an AT to support independent living. Home users were able to complete 61 and $72 \%$ of tasks set for them over the 6 weeks. Satisfaction with the system was strongly linked to the systems responsiveness throughout the evaluation on the BCI satisfaction scale. Additionally, both home users were satisfied with the BCI on the eQUEST 2.0.

The system was innovative in hosting a wide range of applications for the user. It was evident from the evaluation that both home users enjoyed the cognitive rehabilitation tasks and the freedom of exploring all the different Web applications. The smaller matrix sizes proved to be more challenging to select, and thus navigating through the menus to access the various applications could present a challenge. It was suggested to implement a pause button to stop the screen changing. It was difficult to use; it needed to be selected twice to implement and selected two more times to enable selections again. Due to the high suppressed selection rate, this required too much effort to operate. Additionally, a delay in the flashing was recommended in applications, such as the web browser and cognitive rehabilitation, to give the user an opportunity to decide on their next selection. The famous faces were a feature that users felt supported their interaction with the BCI and one participant would have liked to use his family members' faces instead of celebrities.

The identification of caregiver's satisfaction was essential because the caregiver is fundamental to the real world deployment of BCI systems; however, there is little evidence of their important role in the literature. Essentially non-experts caregivers were able to support users at home, and it was evident they were very motivated to support the completion of the tasks. The evaluation required extensive commitment from both the end-user and the caregiver due to the time obligations and patience needed when experiencing the technical challenges which is the nature of testing emerging technologies. A number of recommendations from the caregivers will endeavor to make BCI systems easier, such as an even easier set up process, robust equipment for everyday use, and a quicker process to set the end-user up. The evaluation is important to continue bridging the gap between engineer's perspectives of "easy set up" and the non-experts opinion.

\section{CONCLUSION AND LESSONS LEARNT}

In a world that is much more digitally enabled than ever before, people living with physical and cognitive disabilities struggle to

TABLE 2 | The caregivers' satisfaction questionnaire.

\begin{tabular}{|c|c|c|c|c|}
\hline & $\begin{array}{l}\text { Do you feel confident operating } \\
\text { the system without support? }\end{array}$ & $\begin{array}{l}\text { What did you like } \\
\text { about the system? }\end{array}$ & $\begin{array}{l}\text { What do you not like } \\
\text { about the system? }\end{array}$ & $\begin{array}{l}\text { Could anything be done to make this } \\
\text { system easier to use on a regular base }\end{array}$ \\
\hline CG1 & $\begin{array}{l}\text { Yes: "Familiarity and experience } \\
\text { made it easier to deal with any } \\
\text { technical issues as the testing } \\
\text { progressed" }\end{array}$ & $\begin{array}{l}\text { "When users where able to } \\
\text { complete tasks quickly it gave } \\
\text { them great satisfaction" }\end{array}$ & $\begin{array}{l}\text { "It was inconsistent. I would prefer a } \\
\text { system that looks longer to set up but } \\
\text { was more reliable and responded } \\
\text { more quickly during tasks" }\end{array}$ & $\begin{array}{l}\text { "A totally immersible cap would make the } \\
\text { cleaning easier" }\end{array}$ \\
\hline CG2 & $\begin{array}{l}\text { Yes: "As the weeks have went by I } \\
\text { have got more confident and do feel } \\
\text { like I can operate without support" }\end{array}$ & $\begin{array}{l}\text { "Seeing the difference it can } \\
\text { make to someone's life" }\end{array}$ & $\begin{array}{l}\text { "The issues with connection to } \\
\text { amplifier" }\end{array}$ & $\begin{array}{l}\text { "Just a more reliable connection to enable } \\
\text { ease of set up" }\end{array}$ \\
\hline CG3 & Yes & $\begin{array}{l}\text { "The ease of setting and } \\
\text { sense of achievement" }\end{array}$ & $\mathrm{N} / \mathrm{A}$ & $\begin{array}{l}\text { "It is just the time constraint within the unit } \\
\text { makes this difficult" }\end{array}$ \\
\hline CG4 & $\begin{array}{l}\text { No (CG4 caregiver withdrew after } \\
\text { setting up the user on the } \mathrm{BCl} \text { three } \\
\text { times) }\end{array}$ & $\begin{array}{l}\text { "It was very good working } \\
\text { with only using thought" }\end{array}$ & $\begin{array}{l}\text { "I did not like when it did not work, it } \\
\text { could take over } 1 \text { h for the session" }\end{array}$ & $\begin{array}{l}\text { "More training should be given to the } \\
\text { caregiver so problems could be fixed } \\
\text { easily" }\end{array}$ \\
\hline
\end{tabular}


exert autonomy and independence in their own home and beyond. The BackHome system together with the services it is offering to make a significant contribution for people in need to enhance their opportunities for physical and social inclusion and effective assistance. In fact, with the provision of practical electrodes, easyto-use software, and home support tools, BackHome facilitates independent home use of BCI technology, which can already be considered for the first time as an alternative Assistive Technology.

The findings from the project present challenges to pave the way for later future improvements to further bridge the gap between a laboratory-based BCI and an Assistive Technology for home use, as well as improve understanding of brain research. The results have shown that we have reduced the gap between the initial hardware and software laboratory prototypes intended for research and developed a final product composed of a highly modular, scalable, and distributed architecture ready to host a multifeatured BCI; light, wireless, and ergonomic BCI equipment; a one-click setup and training interface for non-expert caregivers; a complete set of fine-tuned services to satisfy most end-users' needs; a telemonitoring and home support system to remotely monitor and assist BCI independent use; and a web portal for remote experts and therapists to assess the use of the system and the habits and quality of life of the individual.

Of course moving BCI technology from laboratories to endusers' homes brought very demanding issues, which still need to be carefully addressed. This first phase of home-based testing of any emerging technology is essential to identify how it can be operated in an uncontrolled environment and the unique experiences of people without expertise setting it up on an ongoing base. However, it is through these lessons and experiences that technology can be refined and made into a useful product. The technological infrastructure needed to support a BCI system at home is important to consider. Our end-users are living within a supervised and supported living environment, which may be common for disabled people who could benefit from the use of $\mathrm{BCI}$ as an alternative Assistive Technology. This is an important lesson as trend grows to develop ambitious technologies to enable people; it is essential that living environments can support the transition of complex technologies into real life settings. Besides those minimum requirements, we will still need to address important improvements raised by end-users and caregivers. For example, the system was reported as very slow, and this was especially annoying when waiting for a change on the BCI mask when a smart home selection was done taking a minute or more because of the interoperation with an external module. So, it is therefore crucial to enhance environmental control through BCI in the future. Implementing all the features of the BackHome system into one single screen instead of two would reduce the hardware necessary and would also make it easier for the user to interact with the system. The robustness of g.Nautilus is an area for future development as within a home environment with non-experts the wires in the cap became fragile and even broke. The esthetic style of the cap could also be improved although becoming wireless was perceived as very satisfactory for the home users. As stated above, refining the dynamic stopping method to accurately execute suppressed selections only when the end-user is not interacting with the BCI matrix, will dramatically improve responsiveness of the system, perceived control by end-users, overall accuracy and performance, and ultimately user satisfaction.

As a matter of fact, results about independent home evaluation of the BackHome system presented in this paper show a good acceptance of the system by both home users and caregivers. We need to acknowledge that although the system has been tested by many able-bodied and disabled users throughout the entire project life-cycle, only two end-users out of four succeeded to test the final system at their own home. The many technical, ethical, and economic logistics needed for such a complex real world evaluation prevented a broader testing. Being promising, the potential socioeconomic impact of the exploitation of the system, as well as barriers and facilitators for future deployment, has been analyzed and reported at the end of the project. In the very near future, a niche segment of end-users, severe disabled people who are not capable to use eye-trackers and other available technologies, will soon have BCI as their Assistive Technology of choice. Backhome is, therefore, a significant achievement in BCI research, specifically linked to the "replace" and "restore" application scenarios.

In a broader perspective, we are investigating how the solution presented in this paper may reach a larger audience (not only BCI users), providing a better assistance and support to people in need. In so doing, some of the components of the BackHome system have the potentiality to provide services of advanced teleassistance, chronic care management, and social participation targeted to the elderly, the chronic patient, and the disabled not necessarily using BCI as the end-user interface. This is a nice lesson learnt of how research in a complex use case requiring multidisciplinary knowledge can pave the path to simpler and easier to market innovation solutions, which may hit the market and help return the investment in research and development.

\section{AUTHOR CONTRIBUTIONS}

All the authors contributed to the definition, revision, and completion of the work. The author, FM, conceived, planned, coordinated, and drove the scientific work toward conclusions and lessons learnt. EV edited the description of materials and methods and supervised the analysis and interpretation of data. XR-P was involved in analysis of data coming from the telemonitoring system. MS deployed the Therapist Station. SD integrated and deployed the telemonitoring system. CG coordinated definition and deployment of BCI system. $\mathrm{CH}$ and $\mathrm{AE}$ worked on the development of g.Nautilus and interaction of BCI with home. HL worked on exploitation of outcomes. SM, EA, and JD conducted the evaluation with the end-users at home for the collection and analysis of results.

\section{ACKNOWLEDGMENTS}

The study was funded by the European Community for research, technological development, and demonstration activities under the Seventh Framework Programme (FP7, 2007-13), project grant agreement number 288566 (BackHome). This paper reflects only the authors' views and funding agencies are not liable for any use that may be made of the information contained herein. 


\section{REFERENCES}

Brunner, C., Birbaumer, N., Blankertz, B., Guger, C., Kübler, A., Mattia, D., et al. (2015). BNCI horizon 2020: towards a roadmap for the BCI community. Brain Comput. Interfaces 2, 1-10. doi:10.1080/2326263X.2015.1008956

Daly, J., Armstrong, E., Thomson, E., Andreas, P., and Martin, S. (2015a). P300 brain computer interface control after an acquired brain injury. Int. J. Recent Innov. Trends Comput. Commun. 3, 318-325. doi:10.17762/ijritcc2321-8169. 150165

Daly, J., Pinegger, A., Armstrong, E., Thomson, E., Gernot, M.-P., and Martin, S. (2015b). In pursuit of an easy to use brain computer interface for domestic use in a population with brain injury. Int. J. Recent Innov. Trends Comput. Commun. 3, 318-325. doi:10.17762/ijritcc2321-8169.150165

Demers, L., Weiss-Lambrou, R., and Ska, B. (2002). The quebec user evaluation of satisfaction with assistive technology (quest 2.0): an overview and recent progress. Technol. Disabil. 14, 101-105. doi:10.13072/midss.298

Hart, S. G., and Staveland, L. E. (1988). Development of NASA-TLX (task load index): results of empirical and theoretical research. Adv. Psychol. 52, 139-183. doi:10.1016/S0166-4115(08)62386-9

Hintermüller, C., Kapeller, C., Edlinger, G., and Guger, C. (2013). BCI Integration: Application Interfaces, Brain-Computer Interface Systems - Recent Progress and Future Prospects. Rijeka: InTech.

Hintermüller, C., Vargiu, E., Halder, S., Daly, J., Miralles, F., Lowish, H., et al. (2015). "Brain neural computer interface for everyday home usage," in Universal Access in Human-Computer Interaction. Access to Interaction, eds M. Antona and C. Stephanidis (Berlin: Springer), 437-446.

Holz, E. M., Botrel, L., Kaufmann, T., and Kübler, A. (2015). Long-term independent brain-computer interface home use improves quality of life of a patient in the locked-in state: a case study. Arch. Phys. Med. Rehabil. 96, S16-S26. doi:10.1016/j.apmr.2014.03.035

Holz, E. M., Höhne, J., Staiger-Sälzer, P., Tangermann, M., and Kübler, A. (2013). Brain-computer interface controlled gaming: evaluation of usability by severely motor restricted end-users. Artif. Intell. Med. 59, 111-120. doi:10.1016/j.artmed. 2013.08.001

Kapeller, C., Hintermuller, C., Abu-Alqumsan, M., Pruckl, R., Peer, A., and Guger, C. (2013). "A BCI using VEP for continuous control of a mobile robot," in Engineering in Medicine and Biology Society (EMBC), 2013 35th Annual International Conference of the IEEE (Osaka: IEEE), 5254-5257.

Käthner, I., Daly, J., Halder, S., Räderscheidt, J., Armstrong, E., Dauwalder, S. et al. (2014). "A p300 BCI for e-inclusion, cognitive rehabilitation and smart home control," in Proceedings of the 6th International Brain-Computer Interface Conference, eds G. R. M.üller-Putz, G. Bauernfeind, C. Brunner, D. Steyrl, S. Wriessnegger, and R. Scherer (Graz: Verlag der Technischen Universität Graz 2014), 60-63.

Kaufmann, T., Schulz, S. M., Köblitz, A., Renner, G., Wessig, C., and Kübler, A. (2013). Face stimuli effectively prevent brain-computer interface inefficiency in patients with neurodegenerative disease. Neurophysiol. Clin. 124, 893-900. doi:10.1016/j.clinph.2012.11.006

Kübler, A., Holz, E. M., Riccio, A., Zickler, C., Kaufmann, T., Kleih, S. C., et al. (2014). The user-centered design as novel perspective for evaluating the usability of BCI-controlled applications. PLoS ONE 9:e112392. doi:10.1371/journal.pone. 0112392
Kübler, A., Müller-Putz, G., and Mattia, D. (2015). User-centred design in braincomputer interface research and development. Ann. Phys. Rehabil. Med. 58, 312-314. doi:10.1016/j.rehab.2015.06.003

Lynch, B. (2002). Historical review of computer-assisted cognitive retraining. J. Head Trauma Rehabil. 17, 446-457. doi:10.1097/00001199-200210000-00006

Miralles, F., Vargiu, E., Dauwalder, S., Solà, M., Müller-Putz, G., Wriessnegger, S. C., et al. (2015). Brain computer interface on track to home. ScientificWorldJournal 2015, 623896. doi:10.1155/2015/623896

Münssinger, J., Halder, S., Kleih, S., Furdea, A., Raco, V., Hösle, A., et al. (2010) Brain painting: first evaluation of a new brain computer interface application with ALS-patients and healthy volunteers. Front. Neurosci. 4:182. doi:10.3389/ fnins.2010.00182

Nijboer, F., Sellers, E., Mellinger, J., Jordan, M., Matuz, T., Furdea, A., et al. (2008) A p300-based brain-computer interface for people with amyotrophic lateral sclerosis. Neurophysiol. Clin. 119, 1909-1916. doi:10.1016/j.clinph.2008.03.034

Rafael-Palou, X., Vargiu, E., and Miralles, F. (2015). "Monitoring people that need assistance through a sensor-based system: evaluation and first results," in Proceedings of AI-AM/NetMed 2015 Artificial Intelligence and Assistive Medicine (AIME 2015), CEUR Workshop Proceedings, Vol. 1389. Pavia.

Schreuder, M., Riccio, A., Risetti, M., Dähne, S., Ramsay, A., Williamson, J., et al. (2013). User-centered design in brain-computer interfaces? A case study. Artif. Intell. Med. 59, 71-80. doi:10.1016/j.artmed.2013.07.005

Sellers, E. W., Vaughan, T. M., and Wolpaw, J. R. (2010). A brain-computer interface for long-term independent home use. Amyotroph. Lateral Scl. 11, 449-455. doi:10.3109/17482961003777470

Vargiu, E., Dauwalder, S., Daly, J., Armstrong, E., Martin, S., and Miralles, F. (2014). "Cognitive rehabilitation through BNCI: serious games in backhome," in Proceedings of the 6th International Brain-Computer Interface Conference, eds G. R. Müller-Putz, G. Bauernfeind, C. Brunner, D. Steyrl, S. Wriessnegger, and R. Scherer (Graz: Verlag der Technischen Universität Graz 2014), 36-39.

Vargiu, E., Rafael-Palou, X., and Miralles, F. (2015). Experimenting quality of life telemonitoring in a real scenario. Artif. Intell. Res. 4, 136-142. doi:10.5430/air. v4n2p136

Vaughan, T. M., McFarland, D. J., Schalk, G., Sarnacki, W., Krusienski, D. J., Sellers, E. W., et al. (2006). The Wadsworth BCI research and development program: at home with BCI. IEEE Trans. Trans. Neural Syst. Rehab. Eng. 14, 229-233. doi:10.1109/TNSRE.2006.875577

Zickler, C., Halder, S., Kleih, S. C., Herbert, C., and Kübler, A. (2013). Brain painting: usability testing according to the user-centered design in end users with severe motor paralysis. Artif. Intell. Med. 59, 99-110. doi:10.1016/j.artmed.2013.08.003

Conflict of Interest Statement: The authors declare that the research was conducted in the absence of any commercial or financial relationships that could be construed as a potential conflict of interest.

Copyright (C) 2015 Miralles, Vargiu, Rafael-Palou, Solà, Dauwalder, Guger, Hintermüller, Espinosa, Lowish, Martin, Armstrong and Daly. This is an open-access article distributed under the terms of the Creative Commons Attribution License (CC BY). The use, distribution or reproduction in other forums is permitted, provided the original author(s) or licensor are credited and that the original publication in this journal is cited, in accordance with accepted academic practice. No use, distribution or reproduction is permitted which does not comply with these terms. 